\title{
Immune checkpoint inhibitors: the battle of giants
}

\author{
"The checkpoint molecule that has been the most extensively studied \\ is PD-1 and its ligand PD-L1. Inhibiting the binding of PD-L1 to PD-1 \\ with an antibody indeed leads to killing of tumor cells by T cells."
}

First draft submitted: 11 April 2017; Accepted for publication: 20 April 2017; Published online: 11 July 2017

Keywords: cancer $\bullet$ infringement $\bullet$ license $\bullet$ litigation $\bullet$ monoclonal antibodies $\bullet$ nivolumab - pembrolizumab • PD-1

The treatment of cancer is facing a revolution with the appearance of immune checkpoint inhibitors on the market. Immune checkpoint inhibitors allow the immune system to reactivate against the tumor by inhibiting checkpoint molecules on $\mathrm{T}$ cells. The checkpoint molecule that has been the most extensively studied is PD-1 and its ligand PD-L1. Inhibiting the binding of PD-L1 to PD-1 with an antibody indeed leads to killing of tumor cells by T cells. Currently, there are two approved drugs for PD-1, in other words, nivolumab (Opdivo ${ }^{\circledR}$, BristolMyers Squibb, NY, USA) and pembrolizumab (Keytruda ${ }^{\circledR}$, Merck \& Co, NJ, USA), which are human and humanized IgG4 monoclonal antibodies, respectively. Nivolumab was the first PD-1 inhibitor to be approved in July 2014. It has been approved for the treatment of advanced melanoma, advanced renal cell carcinoma, Hodgkin lymphoma, non-small-cell lung cancer (NSCLC) and metastatic melanoma. Pembrolizumab has been approved for the treatment of advanced melanoma, NSCLC and metastatic melanoma. It was estimated that PD-1 inhibitors brought the sales of US $\$ 35$ billion. Nivolumab had US\$2.1 billion sales versus pembrolizumab's US\$566 million in 2015. PD-1 inhibitors have replaced existing chemotherapy and radiotherapy for treating cancer. Several clinical trials for combination therapy of these inhibitors are ongoing with more than 200 active clinical trials.

\section{Patents}

Bristol-Myers Squibb (BMS) and Ono are the current co-applicants of the patent family relating to nivolumab. EP2161336 was granted on 31 July 2013 with two main independent claims. Claim 1 was related to an isolated monoclonal antibody that specifically binds to human PD-1 and having heavy and light chain variable regions characterized by the specific complementarity-determining region sequences of nivolumab [1]. Claim 3 was related to a monoclonal antibody, which cross-competes for binding to PD-1 with the antibody of claim 1. The claim was finally canceled during the opposition lodged by Merck so as to counteract the novelty objection. Recently, the opposition division decided on 2 March 2017 to maintain the patent in amended form with first independent claims relating to an isolated human antibody characterized by the heavy and light variables regions of nivolumab. The corresponding US patent US8779105 was granted on 15 July 2014 with an independent claim relating to a

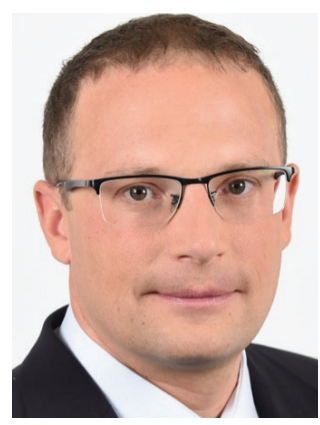

Matthieu Collin

Intellectual Property Director, INSERM Transfert, 7 Rue Watt, Paris 75013, France

matthieu.collin@inserm-transfert.fr newlands press part of 
monoclonal antibody or an antigen-binding portion thereof, which cross-competes for binding to PD-1 with a reference antibody or antigen-binding portion thereof that comprises among others the heavy and light variables regions of nivolumab [2]. Nivolumab is specifically the subject matter of claim 3. Patent rights will expire on 2 May 2026 excepting for the USA, wherein the term was extended to 487 days by United States Patent and Trademark Office.

Pembrolizumab was developed by Carven and colleagues at Organon, the healthcare section of Akzo Nobel, Organon was acquired by Schering-Plough in November 2007. Schering-Plough was then taken over by Merck in November 2009. EP2170959 was granted on 2 October 2013 with a first independent claim relating to an antibody or antibody fragment which binds to human PD-1, comprising the complementarity-determining region of pembrolizumab [3]. No opposition was filed against the European patent. US8354509 was granted on 15 January 2013 with a similar independent claim as for European patent [4]. The patent rights will expire on 13 June 2028.

\section{"Finally, on 20 January 2017, Merck, Bristol-Myers Squibb and Ono finally announced that the companies have signed a global patent license agreement.}

\section{Patent conflicts}

In September 2014, Merck was sued by BMS and Ono for patent infringement before the Delaware District Court [5]. The claimants alleged that commercialization of pembrolizumab infringes US Patent US8728474, which has a first independent claim relating to a method for treatment of a tumor comprising administering an anti-PD-1 monoclonal antibody [6]. Interestingly, the claimants did not seek for an injunction, but so far only demanded damages. Later, in July 2015, BMS and Ono also lodged a suit based in just granted US Patent US9073994, having a first claim relating to method of treating a metastatic melanoma comprising intravenously administering an effective amount a human or humanized anti-PD-1 monoclonal antibody [7].

In April 2014, Merck filed a notice of opposition against BMS and Ono's European EP1537878 having a main claim, which relates to the use of an anti-PD-1 antibody for the manufacture of a medicament for cancer treatment [8]. The opposition was finally rejected by the European Patent Office, deciding that the claims were novel. In May 2014, Merck sued Ono and BMS in the UK for invalidity of the UK parts of EP1537878 and EP2161336. In reply to the injunction, Ono and
BMS filed an action for a declaratory judgment that the EP1537878 patent would be infringed by the commercialization of pembrolizumab in the UK [9]. Still in the UK, Merck finally filed a request for declaration of noninfringement with respect to EP2161336 patent [9].

Finally, on 20 January 2017, Merck, BMS and Ono finally announced that the companies have signed a global patent license agreement [10]. According to the agreement, Merck shall make an upfront payment of US $\$ 625$ million to BMS and Ono and is also obligated to pay degressive royalties on global sales of pembrolizumab $(6.5-2.5 \%)$. The payments will be shared between BMS and Ono in a 75/25\% allocation, respectively. Under the agreement, the companies have also granted cross-licenses under their respective patent portfolios and decided to put an end to all patent-infringement litigations and conflicts.

\section{Conclusion}

In conclusion, two observations can be made. First of all, from a legal point of view, with the withdrawal of claim 3 during opposition proceedings of EP2161336, we lose the opportunity to get an ex officio clarification regarding patent claims reciting "antibodies that compete for binding with a reference antibody." This frequent type of claim in the field of antibodies indeed bears uncertainty of interpretation since clarity is potentially highly challengeable. Position of European Patent Office would have certainly served the interest of legal certainty. Second, the patent license agreement reached between BMS and Merck on pembrolizumab represents probably just a pause in the course of patent and commercialization battles in the field of immune checkpoint inhibitors. The pipeline of PD-1 and PD-L1 is absolutely profuse with approximately 47 drugs with 35 companies involved, including big pharmaceutical companies. For instance, atezolizumab (Tecentriq ${ }^{\circledR}$, Genentech, CA, USA), which is a fully humanized, engineered monoclonal antibody of IgG1 isotype against the protein PD-L1, was recently approved by the US FDA for the treatment of patients with metastatic NSCLC [11] and is currently in clinical trials as an immunotherapy for several other types of solid tumors. BMS will probably consider that the antibody developed by Roche would infringe US9402899, which was granted with a first independent claim reciting a method of treating a tumor comprising administering to the human an effective amount of an anti-PD-L1 monoclonal antibody that inhibits an interaction between PD-1 and PD-L1 [12].

Financial \& competing interests disclosure

The author has no relevant affiliations or financial involvement with any organization or entity with a financial interest 
in or financial conflict with the subject matter or materials discussed in the manuscript. This includes employment, consultancies, honoraria, stock ownership or options, expert

\section{References}

1 ONO Pharmaceutical Co., Ltd., Medarex Inc. EP2161336 (2013).

2 Medarex LLC. US8779105 (2014).

3 Merck Sharp \& Dohme BV. EP2170959 (2013).

4 Msd Oss BV. US8354509 (2013).

5 BMS \& Ono v. Merck \& Co., case 1:14-cv-01131, complaint filed (2014).

6 Ono Pharmaceutical Co., Ltd. Tasuku Honjo US8728474 (2014).

7 Ono Pharmaceutical Co., Ltd. Tasuku Honjo US9073994 (2015). testimony, grants or patents received or pending, or royalties. No writing assistance was utilized in the production of this manuscript.

8 Ono Pharmaceutical Co., Ltd. Tasuku Honjo EP1537878 (2010).

9 Storz U. Intellectual property issues of immune checkpoint inhibitors. $m A b s$ 8, 10-26 (2016).

$10 \quad$ BMS press release 20 January 2017. https://news.bms.com/

11 Roche Press Release 19 October 2016. www.roche.com/media/store/releases.htm

12 ONO Pharmaceutical Co., Ltd. Tasuku Honjo US9402899 (2016). 